\title{
Neuroradiologie: ein unterschätztes Fachgebiet
}

\author{
Anton Valavanisa, Luca Remonda ${ }^{b}$, Jan Grallac

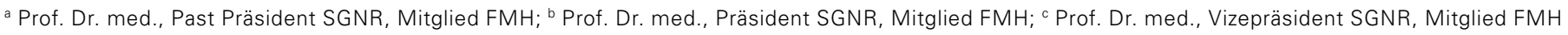

Seit fast 15 Jahren führt die Schweizerische Gesellschaft für Neuroradiologie (SGNR) ihre Kongresse zusammen mit Fachgesellschaften durch, die sich einer verwandten Neurodisziplin widmen. So organisierte die SGNR ihren diesjährigen Kongress Anfang Juni gemeinsam mit der Schweizerischen Gesellschaft für Neurochirurgie (SGNC).

Schon diese etablierte Interdisziplinarität der jährlichen Fortbildungsveranstaltungen zeigt auf, wie nahe sich die Disziplinen innerhalb der klinischen Neurowissenschaften stehen und wie stark sie sowohl in der Praxis als auch in der Theorie aufeinander angewiesen sind. Doch obwohl ein harmonisches Zusammenspiel zwischen der Neuroradiologie und den anderen klinischen Neurowissenschaften für die erfolgreiche Behandlung vieler neurologischer Krankheiten unverzichtbar ist, wird der Neuroradiologie nicht jene Verantwortung zuteil, die heute eigentlich erforderlich wäre. Denn im Gegensatz zur Neurologie und Neurochirurgie verfügt die Neuroradiologie über keinen eigenen Facharzttitel. Das Ungleichgewicht, welches dadurch innerhalb dieser drei Kerndisziplinen der klinischen Neurowissenschaften entsteht, gefährdet zunehmend die Behandlungsqualität.

\section{Der schnelle Aufstieg einer jungen Disziplin}

Als relativ junges Fachgebiet entstand die heutige Neuroradiologie in den 50ern und 60ern des vergangenen Jahrhunderts. Im Gegensatz zur gängigen Auffassung, dass die Disziplin aus der medizinischen Radiologie hervorgegangen ist, entwickelte sich die Neuroradiologie inhaltlich und technisch aus Pionierleistungen der Neurochirurgie und der Neurologie. Eine methodologische Nähe zur Radiologie existiert, weil sich beide Fachgebiete ähnlicher bildgebender Methoden bedienen. Und wie die medizinische Radiologie beschäftigte sich die Neuroradiologie anfangs ausschliesslich mit diagnostischen Fragen. Ende der 1960er Jahre kam durch endovaskuläre Kathetertechniken die Therapie unterschiedlicher Krankheitsbilder hinzu, wodurch das
Aufgabengebiet der Neuroradiologie sich ausweitete und diese einen festen Platz innerhalb der klinischen Neurowissenschaften einnahm. 1989 wurde dem zunehmenden Bedürfnis nach standespolitischer Vertretung entsprochen und eine eigene Fachgesellschaft gegründet. Die Schweizerische Gesellschaft für Neuroradiologie (SGNR) hat heute über 180 Mitglieder.

\section{Schwerpunkttitel: nicht mehr ausreichend}

1991 war die Schweiz das erste Land Europas, welches die Bedeutung der Disziplin mit dem Untertitel FMH für Neuroradiologie anerkannte. Gleichzeitig begann in der Schweiz der Aufbau mehrerer neuroradiologischer Kliniken. Im Jahr 2000 folgte dann die Schaffung zweier Schwerpunkte - einer für diagnostische und einer für invasive Neuroradiologie. Diese bestehen bis heute, obwohl sich seither das Fach selber, aber auch die Rahmenbedingungen, markant verändert haben. Die Bevölkerung der Schweiz wächst stetig und die Lebenserwartung steigt. Deshalb ist mit einer Zunahme neurologischer Erkrankungen und folglich auch mit einem höheren Bedarf an neuroradiologischen Leistungen zu rechnen. Dennoch steht eine Anpassung neuroradiologischer Arbeitsbedingungen an die heutigen Anforderungen aus.

Pro Woche erleiden 400 bis 500 Personen in der Schweiz einen zerebralen Schlaganfall. Dazu kommen wöchentlich 10 bis 15 Personen, bei denen ein Hirnaneurysma reisst. Ein Drittel der Patienten stirbt an diesen Erkrankungen. Dennoch steigt die Überlebenschance stetig an, was zu einem grossen Teil der guten Zusammenarbeit zwischen Neurologen, Neurochirurgen und eben Neuroradiologen zu verdanken ist, die Betroffene in sogenannten Stroke Centers oder kleineren Stroke 
Units schnellstmöglich behandeln. Dabei sind Neuroradiologen und Neuroradiologinnen in den meisten Fällen viel mehr als Navigatoren, die andere Fachleute durch das Nervensystem führen: Sämtliche interventionellen Behandlungen von Schlaganfällen und rupturierten Aneurysmen im Gehirn werden gegenwärtig von interventionellen Neuroradiologen selbst durchgeführt.

\section{Massive Unterversorgung}

In der Schweiz gibt es momentan zehn solche Stroke Centers. Die Richtlinien dieser hochspezialisierten Kompetenzzentren legen fest, dass an 365 Tagen rund um die Uhr kompetente Neuroradiologen zur Stelle sein müssen, um so schnell und effizient wie möglich eingreifen zu können. Bei 25 Ärztinnen und Ärzten, die sich in der Schweiz vollamtlich der interventionellen Neuroradiologie widmen, ist der eigentliche Bedarf von 40 Neuroradiologen alleine in den zehn Stroke Centers bereits heute nicht annähernd gedeckt. In solchen Fällen des Fachkräftemangels bedient sich das schweizerische Gesundheitssystem gerne der Rekrutierung aus dem Ausland, doch das ist selbst mittelfristig keine nachhaltige Lösung.

Doch wie kommt es überhaupt zu diesem Mangel an Neuroradiologen? Einer der Hauptgründe dafür ist sicherlich die Länge der Weiterbildung. Junge Assistenzärzte, die eine Karriere in der Neuroradiologie anstreben, müssen zunächst den Facharzt in Radiologie erwerben; das dauert durchschnittlich fünf, nicht selten sechs Jahre, von denen einige für die Aneignung von Wissen verwendet werden, das kein Neuroradiologe später in seiner Tätigkeit benötigt. Erst im Anschluss können die Schwerpunkttitel diagnostische und invasive Neuroradiologie erworben werden. Insgesamt dauert die Weiterbildung zum interventionellen Neuroradiologen acht bis neun Jahre. Das schreckt potenzielle Talente ab und macht die Neuroradiologie für möglichen Nachwuchs verständlicherweise unattraktiv. Wer zudem die Weiterbildungsvolumina der Neuroradiologie und der Radiologie miteinander vergleicht, wird feststellen, dass deren Umfang und Gehalt ähnlich ist. Da den beiden Schwerpunkttiteln für Neuroradiologie aber nicht sechs, sondern nur zwei bis drei Jahre zur Verfügung stehen, kann nicht einmal das gesamte Curriculum vermittelt werden. Wo die Radiologie also zu viel Zeit bekommt, erhält die Neuroradiologie mit ihren beiden Schwerpunkten zu wenig.

\section{Eine einfache Lösung}

Die einzig zielführende Lösung für die genannten Probleme ist die Einführung eines Facharzttitels in Neuroradiologie. Dadurch hätten angehende Neuroradiologen zum einen sechs Jahre Zeit um alles zu lernen, was sie wissen sollten, ohne wertvolle Zeit mit der Aneignung von Kenntnissen zu verbringen, die im neuroradiologischen Alltag keine Relevanz besitzen. Die Weiterbildung zum Neuroradiologen wäre kürzer und gleichzeitig gründlicher, die Attraktivität der Disziplin würde, wie auch der klinische Bezug, steigen und der Bedarf an Fachkräften wäre innert einiger Jahre gedeckt.

Das jahrzehntelang vorherrschende Ungleichgewicht zwischen der Neurologie, der Neurochirurgie und der Neuroradiologie würde mit der Schaffung eines Facharzttitels Neuroradiologie behoben, was nicht nur der Neuroradiologie zugutekommen würde, sondern den gesamten klinischen Neurowissenschaften, wenn nicht sogar dem gesamten Gesundheitssystem: Der Facharzttitel würde allen klinischen Neurowissenschaftlern in Weiterbildung eine Rotation in andere, nun auch standespolitisch gleichgestellte Neurofächer ermöglichen, Interdisziplinarität würde ausgebaut. Die Folge wären ein markanter Qualitätsanstieg und höhere Sicherheit bei gleichzeitigen Kosteneinsparungen, weil die Anzahl Zweit- oder sogar Drittuntersuchungen reduziert würde.

\section{Grundlose Angst}

Die Befürchtung einiger, dass mit der Schaffung des Facharztes Neuroradiologie die Kosten im hiesigen Gesundheitswesen explodieren würden, sind unbegründet. Der Leistungskatalog der Neuroradiologie bliebe unverändert, es würden keine neuen Kliniken oder Einrichtungen für Neuroradiologie gebaut und strukturelle Änderungen in den Spitälern wären ebenfalls keine nötig. Wie die Nuklearmedizin und die Radioonkologie würde sich auch die Neuroradiologie mit der Einführung eines Facharzttitels von der Muttergesellschaft trennen. Doch die infrastrukturelle Anbindung an die Radiologie bliebe auch nach der Einführung des Titels bestehen Natürlich würde auch die Weiterbildung in allgemeiner diagnostischer Neuroradiologie für angehende Radiologen mit dem Facharzttitel nicht wegfallen.

Die Etablierung eines Facharzttitels in Neuroradiologie ist längst notwendig - für die Gewährleistung der Behandlungsqualität und für die Patientensicherheit. 